\title{
Nonadiabatic Noncyclic Geometric Phase and Ensemble Average Spectrum of Conductance in Disordered Mesoscopic Rings with Spin-Orbit Coupling
}

\author{
Shi-Liang Zhu and Z. D. Wang* \\ Department of Physics, University of Hong Kong, \\ Pokfulam Road, Hong Kong, China
}

(Received 1 October 1999)

\begin{abstract}
We generalize Yang's theory from the $U(1)$ gauge field to the non-Abelian $U(1) \times S U(2)_{\text {spin }}$ gauge field. Based on this generalization and taking into account the geometric Pancharatnam phase as well as an effective Aharonov-Bohm (AB) phase in nonadiabatic noncyclic transport, we calculate the ensemble average Fourier spectrum of the conductance in disordered mesoscopic rings connected to two leads. Our theory can explain the experimental results reported by Morpurgo et al. [Phys. Rev. Lett. 80, 1050 (1998)] more satisfactorily. We indicate that the observed splitting stems from the nonadiabatic noncyclic Pancharatnam phase and the effective $\mathrm{AB}$ phase, both being dependent on spin-orbit coupling.
\end{abstract}

PACS numbers: 73.23.-b, 03.65.Bz, 71.70.Ej

As is well known, the geometric phase [1-4] has manifested itself extensively in physics, particularly in mesoscopic systems where quantum interference is extremely important [5-9]. Recently, Morpurgo et al. [10] reported a novel splitting of the main peak [corresponding to the $h c / e$ Aharonov-Bohm (AB) oscillations] in the ensemble average Fourier spectrum of the conductance in open mesoscopic rings [11]. The authors conjectured that the observed splitting is due to the spin-orbit (SO)-induced Berry's phase [6-9]. It is probably strong experimental evidence showing an important effect of the SO geometric phase on quantum transport. Although it was concluded that, in a mesoscopic ring possessing the time-reversal symmetry in the absence of $\mathrm{AB}$ flux, the SO-dependent transport can be treated formally in the absence of SO coupling, but with an effective magnetic flux [5], it is unclear yet how to calculate the value of this flux, as well as the ensemble average spectrum of conductance; besides, it is not clear either whether the above conclusion is still valid in the presence of an arbitrary local magnetic field on the ring (i.e., the aforementioned time-reversal symmetry is broken), which appears to be the experimental case in Ref. [10]. Mal'shukov et al. attempted to account for the observed splitting of the main peak but were not quite successful [12]. Three aspects of the experiment require a theoretical explanation. (i) The magnitude of the observed splitting is surprisingly large when compared with an estimation based on the adiabatic approximation in a clean mesoscopic ring. For a clean ring with radius $r$ subject to a crown-shaped effective magnetic field $\mathbf{B}_{\text {eff }}=$ $\left(B_{0} \cos \varphi_{r}, B_{0} \sin \varphi_{r}, B_{z}\right)$ in the cyclindrical coordinates, Stern [7] predicted that the inverse $B_{z}$ period becomes $\left(\Delta B_{z}\right)^{-1}=\pi r^{2} / \phi_{0} \pm 1 /\left(2 B_{0}\right)$ at $B_{0} \gg B_{z}$, and the splitting would be $\sim 1.2 \times 10^{-3} \mathrm{mT}^{-1}$ for an estimated experimental parameter $B_{0} \sim 0.8 \mathrm{~T}$, which is at least 1 order of magnitude less than the observed value. (ii) The origin of the side structure on the main peak needs to be clarified. (iii) Most existing theoretical estimations are crucially based on the adiabatic or cyclic condition; however, neither adiabatic nor cyclic evolution is well satisfied in the experiment $[7,9,13]$. In view of these facts, we believe that the nonadiabatic noncyclic geometric phase, essentially similar to the effective flux addressed formally in Ref. [5] including the nonadiabatic case and being at the same microscopic level as the present work on the treatment of SO, could play a crucial role in the system. This is the key point of the present work, being essentially different from some existing theoretical analyses [6-9]. However, it is still highly nontrival to evaluate the SOinduced geometric phase in the nonadiabatic noncyclic transport and its effect on the ensemble average spectrum.

It is worth pointing out that the relevant geometric phase detected in the experiment is likely induced by a $U(1) \times$ $\mathrm{SU}(2)_{\text {spin }}$ field. In exploring the global geometrical connotations of gauge fields of either the Abelian U(1) type or the non-Abelian monopole type, Yang et al. showed that the nonintegrable gauge phase factor in the wave function gives an intrinsic and complete description of the relevant field [14], hereafter referred to as Yang's theory. In this Letter, we first generalize Yang's theory to the nonAbelian $\mathrm{U}(1) \times \mathrm{SU}(2)_{\text {spin }}$ electromagnetic field. Using a simple one-dimensional (1D) continuum model for a quasi-1D mesoscopic ring, we then analyze carefully the nonintegrable phase induced by this field and evaluate its effect on the splitting of the main peak in the ensemble average spectrum of the conductance. Remarkably, we find that the splitting as well as the side structure of the main peak stems from the nonadiabatic noncyclic geometric phase.

We consider an electron subject to an electromagnetic field. The corresponding Hamiltonian with $U(1)_{\text {e.m. }} \times$ $\mathrm{SU}(2)_{\text {spin }}$ gauge symmetry is given by [15]

$$
\hat{H}=\frac{1}{2 m}\left(\mathbf{p}+\frac{e}{c} \mathbf{A}-\frac{\mu}{c} \mathbf{a}\right)^{2}-e A^{0}+\mu a^{0}+V(\mathbf{r}),
$$


where $\mu=g \mu_{B} / 2$ with $g$ the gyromagnetic ratio and $\mu_{B}=e \hbar /(2 m c)$ the Bohr magneton. Here $A^{\nu}=\left(A^{0}, \mathbf{A}\right)$ represents a $\mathrm{U}(1)_{\text {e.m. }}$. electromagnetic potential, and $a^{\nu}=$ $\left(a^{0}, \mathbf{a}\right)=(-\vec{\sigma} \cdot \mathbf{B}, \vec{\sigma} \times \mathbf{E} / 2)$ is an $\mathrm{SU}(2)_{\text {spin }}$ potential with $\vec{\sigma}$ denoting the Pauli matrix. $V(\mathbf{r})$ is an arbitrary spinindependent local potential at the point $\mathbf{r}$. The Schrödinger equation for the normalized two-component wave function $\Psi\left(x^{\nu}\right)$ reads $i \hbar \partial_{t} \Psi\left(x^{\nu}\right)=\hat{H} \Psi\left(x^{\nu}\right)$. By introducing a new wave function [15], $\Psi_{0}\left(x^{\nu}\right)=\hat{U} \Psi\left(x^{\nu}\right)$, where

$$
\hat{U}=\exp \left(i \frac{e}{\hbar c} \int_{\Gamma} A_{\nu} d x^{\nu}\right) \hat{P} \exp \left(i \frac{\mu}{\hbar c} \int_{\Gamma} a_{\nu} d x^{\nu}\right)
$$

with $\hat{P}$ the path ordering operator and $\Gamma$ an integration curve from a fixed $x_{0}^{\nu}$ to $x^{\nu}$, we find that the Schrödinger equation reduces exactly to $i \hbar \partial_{t} \Psi_{0}\left(x^{\nu}\right)=\hat{H}_{0} \Psi_{0}\left(x^{\nu}\right)$ with

$$
\hat{H}_{0}=\hat{U}\left(\hat{H}-i \hbar \frac{\partial}{\partial t}\right) \hat{U}^{-1}=\frac{(-i \hbar \nabla)^{2}}{2 m}+V(\mathbf{r}) .
$$

Clearly, $\hat{U}$ is a continuous local gauge transformation. Under this gauge transformation, the Hamiltonian (1) is transformed to a Hamiltonian devoid of electromagnetic fields, but with a phase shift in the wave function as seen in Eq. (2). In this sense, the gauge factor in Eq. (2) is just the nonintegrable phase in Yang's theory, which can describe completely the $\mathrm{U}(1) \times \mathrm{SU}(2)_{\text {spin }}$ electromagnetic field. For a mesoscopic ring where the phase memory is retained by electrons, we may conclude that physical properties of the system in the presence of an electromagnetic field can be expressed in terms of the same quantity in the absence of the electromagnetic field, but with a nonintegrable phase being taken into account. An important application is related to the SO coupling: any spin-independent transport quantity can be expressed in terms of the same quantity in the absence of SO scattering but with an effective magnetic flux, an important fact which was observed in Ref. [5] by employing $2 \times 2$ scattering matrices within the framework of a tight-binding model for a mesoscopic ring possessing the time-reversal symmetry in the absence of AB flux. In fact, with the help of this generalized theory, we are able to study a disordered mesoscopic system subject to an electromagnetic field in a simpler way.

We now focus on the phase factor first. To capture essential physics of geometric phase in the present quasi-1D system, we employ a simple 1D model. For a closed path parametrized by arc length $s$, the total phase factor in Eq. (2) is $\gamma_{t}=\gamma_{\mathrm{AB}}+\tilde{\gamma}$, where $\gamma_{\mathrm{AB}}=2 \pi \phi / \phi_{0}$ is the usual $\mathrm{AB}$ phase with $\phi$ the magnetic flux and $\phi_{0}=h c / e$, and $\tilde{\gamma}$ is the second phase factor in Eq. (2), which is determined by a Schrödinger-type equation $[9,16]$

$$
i \hbar \frac{\partial}{\partial s}|\xi(s)\rangle=-\mu \vec{\sigma} \cdot\left(\frac{1}{v} \mathbf{B}-\frac{1}{2 c} \hat{\mathbf{v}} \times \mathbf{E}\right)|\xi(s)\rangle .
$$

Here $\hat{\mathbf{v}}$ is a unit vector along the direction of the velocity $\mathbf{v}=v \hat{\mathbf{v}}$ and $d s=v d t$. Equation (4) describes the evolution of the spin state $|\xi\rangle$ governed by the operator $\hat{U}$.
The phase associated with Eq. (4) can be further written as $\tilde{\gamma}=\gamma_{d}+\gamma_{\mathrm{AB}}^{\text {eff }}+\gamma_{p}$ [3] with the dynamical phase $\gamma_{d}=$ $\mu / \hbar \int\langle\xi(s)|\vec{\sigma} \cdot \mathbf{B} / v| \xi(s)\rangle d s$, the effective AB phase

$$
\gamma_{\mathrm{AB}}^{\text {eff }}=-\frac{\mu}{\hbar} \int\left\langle\xi(s)\left|\frac{1}{2 c} \vec{\sigma} \cdot(\hat{\mathbf{v}} \times \mathbf{E})\right| \xi(s)\right\rangle d s,
$$

and $\gamma_{p}$ is the Pancharatnam phase, to be addressed in detail later. Here we emphasize that $\gamma_{\mathrm{AB}}^{\text {eff }}$ is a kind of geometric phase, though it seems from Eq. (5) as if it were a dynamical phase related to an "effective magnetic field" $-\mathbf{v} \times \mathbf{E} / 2 c$. The reason lies in the fact that the two waves propagating in opposite directions in the ring acquire phases with the opposite sign for $\gamma_{\mathrm{AB}}^{\text {eff }}$ (simply because it depends on the velocity direction $\hat{\mathbf{v}}$ ), but the same sign for $\gamma_{d}$ [17]. The geometrical feature of $\gamma_{\mathrm{AB}}^{\text {eff }}$ seems to be ignored in some earlier analyses [8,9], which appears to be a minor reason for the existing discrepancy between theory and experiment. In fact, $\gamma_{\mathrm{AB}}^{\text {eff }}$ is just induced by an $\mathrm{SU}(2)_{\text {spin }}$ vector potential a, and it is clear from Eq. (1) that a plays a role similar to that of the $\mathrm{U}(1)_{\text {e.m. }}$. vector potential $\mathbf{A}$ in the $\mathrm{AB}$ effect. As a result, it is expected that an effective $\mathrm{AB}$ effect can be induced by this $\mathrm{SU}(2)_{\text {spin }}$ vector potential [13], as was also shown by Choi et al. [18].

For a unit vector $\mathbf{n}=\left(n_{1}, n_{2}, n_{3}\right)=(\sin \theta \cos \varphi$, $\sin \theta \sin \varphi, \cos \theta)$ with $\mathbf{n} \in$ a unit sphere $S^{2}$, each $\mathbf{n}$ corresponds to the spin state $|\xi\rangle=\left[e^{-i \varphi / 2} \cos (\theta / 2)\right.$, $\left.e^{i \varphi / 2} \sin (\theta / 2)\right]^{T}$ via the relation $\mathbf{n}=\langle\xi|\vec{\sigma}| \xi\rangle$, where $T$ represents matrix transposition. The noncyclic Pancharatnam phase accumulated in an evolution of $\mathbf{n}$ is found to be $\gamma_{p}=-\frac{1}{2} \oint_{\partial \Sigma=C} \mathbf{n} \cdot d \mathbf{\Sigma}$ [19], where $d \mathbf{\Sigma}$ is an area element on $S^{2}$, and $C$ is a specific closed curve on $S^{2}$, which is along the actual path of $\mathbf{n}(s)$ plus the shorter geodesic curve from the final point $\mathbf{n}\left(s_{f}\right)=$ $\left(\sin \theta_{f} \cos \varphi_{f}, \sin \theta_{f} \sin \varphi_{f}, \cos \theta_{f}\right)$ to the initial point $\mathbf{n}(0)=\left(\sin \theta_{i} \cos \varphi_{i}, \sin \theta_{i} \sin \varphi_{i}, \cos \theta_{i}\right)$. This Pancharatnam phase can be derived as [19]

$$
\begin{aligned}
\gamma_{p}= & -\frac{1}{2} \int_{0}^{t_{f}} \frac{n_{1} \dot{n}_{2}-n_{2} \dot{n}_{1}}{1+n_{3}} d t \\
& +\arctan \frac{\sin \left(\varphi_{f}-\varphi_{i}\right)}{\cot \frac{\theta_{f}}{2} \cot \frac{\theta_{i}}{2}+\cos \left(\varphi_{f}-\varphi_{i}\right)},
\end{aligned}
$$

where $t_{f}$ is the final time, $\dot{n}_{1,2}=d n_{1,2} / d t$, and $\mathbf{n}$ is determined by the equation

$$
\frac{d \mathbf{n}}{d t}=-\frac{2 \mu}{\hbar}\left(\mathbf{B}-\frac{1}{2 c} \mathbf{v} \times \mathbf{E}\right) \times \mathbf{n},
$$

which represents a spin- $-\frac{1}{2}$ particle moving in an effective magnetic field $(\mathbf{B}-\mathbf{v} \times \mathbf{E} / 2 c)$. This phase is not equal to the cyclic Aharonov-Anandan (AA) phase in general [3], but recovers the AA phase $\gamma_{\mathrm{AA}}=-\frac{1}{2} \int_{0}^{\tau} d t\left(n_{1} \dot{n}_{2}-\right.$ $\left.n_{2} \dot{n}_{1}\right) /\left(1+n_{3}\right)$ for any cyclic evolution with the period $\tau$ [19]. It is remarkable that the nonintegrable phase in Eq. (2) can be evaluated by simply computing Eqs. (5) and (6), while it is hard to calculate the value of the effective flux addressed formally in Ref. [5], particularly in the presence of an arbitrary local magnetic field. 
At this stage, we consider a ring with the Rashba SO interaction (equivalent to an internal electric field $\mathbf{E}=$ $E \mathbf{e}_{z}$ ), subject to a local magnetic field $\mathbf{B}=B_{z} \mathbf{e}_{z}$ and a magnetic flux $\phi=\pi r^{2} B_{z}$. The Hamiltonian, which is in the form of Eq. (1), becomes $[9,13]$

$$
\begin{aligned}
\hat{H}= & \hbar \omega_{r}\left[-i \frac{\partial}{\partial \varphi_{r}}+\frac{\phi}{\phi_{0}}-\frac{\eta}{2}\left(\sigma_{x} \cos \varphi_{r}+\sigma_{y} \sin \varphi_{r}\right)\right]^{2} \\
& -\mu B_{z} \sigma_{z}+V\left(\varphi_{r}\right),
\end{aligned}
$$

where $\omega_{r}=\hbar /\left(2 m r^{2}\right), \varphi_{r}$ is the polar angle, and the normalized electric field strength $\eta=\mu_{B} E r / c \hbar=2 m \kappa r$ with the SO coefficient $\hbar^{2} \kappa$.

We now investigate the electronic transmission across a disordered ring connected to external current leads, schematically illustrated in Fig. 3 in Ref. [20]. In such a system, the electronic transmission is significantly affected by the nonintegrable phase. Using the method originally proposed by Büttiker et al. [20] and our generalization of Yang's theory, the transmission coefficient across the ring is found to be

$$
T_{g}=\frac{\epsilon^{2}}{b^{4}}\left|(b-a, 1) \tilde{T}_{+}\left[\frac{e^{-i \Delta \gamma}}{b^{2}}\left(\begin{array}{cc}
\left(b^{2}-a^{2}\right) & a \\
-a & 1
\end{array}\right) \tilde{T}_{-}\left(\begin{array}{cc}
\left(b^{2}-a^{2}\right) & a \\
-a & 1
\end{array}\right) \tilde{T}_{+}-\tilde{1}\right]^{-1}\left(\begin{array}{c}
b-a \\
-1
\end{array}\right)\right|^{2},
$$

where $\tilde{T}_{+}$and $\tilde{T}_{-}$are the transfer matrices of the upper and lower branches of the ring, $\tilde{1}$ is the unit matrix, $a=$ $\pm(\sqrt{1-2 \epsilon}-1) / 2$, and $b= \pm(\sqrt{1-2 \epsilon}+1) / 2$ with $0 \leq \epsilon \leq 1 / 2 . \Delta \gamma=\gamma_{\mathrm{AB}}+\gamma_{\mathrm{AB}}^{\text {eff }}(\mathbf{n}(0))+\gamma_{p}(\mathbf{n}(0))$ represents the nonadiabatic noncyclic geometrical phase accumulated in the evolution when the electron [with the initial spinstate $\mathbf{n}(0)$ ] moves one cycle in the clockwise sense. For a beam of electron waves with Fermi wave vector $k_{f}$, the rate for electrons to traverse one round in the ring is $\omega_{f}=\hbar k_{f} /(m r)$ for ballistic motion but is estimated approximately to be $\omega_{d}=l \omega_{f} /(2 \pi r)$ for weak diffusive motion [21], where $l$ is the electron mean free path. This rate can be regarded as the angular frequency of the otherwise rotating magnetic field felt by the electron spin [21], which is given by $\mathbf{B}_{\mathrm{eff}}(t)=\left(B_{0}^{f, d} \cos \omega_{f, d} t, B_{0}^{f, d} \sin \omega_{f, d} t, B_{z}\right)$ with $B_{0}^{f, d}=-\eta \hbar \omega_{f, d} / 2 \mu$. Then from the equation $d \mathbf{n}(t) / d t=$ $-(2 \mu / \hbar) \mathbf{B}_{\text {eff }}(t) \times \mathbf{n}(t), \mathbf{n}(t)$ is derived exactly as

$\mathbf{n}^{T}(t)=\left(\begin{array}{ccc}\cos \omega_{f, d} t & -\sin \omega_{f, d} t & 0 \\ \sin \omega_{f, d} t & \cos \omega_{f, d} t & 0 \\ 0 & 0 & 1\end{array}\right) \times\left(\begin{array}{ccc}\sin ^{2} \chi+\cos ^{2} \chi \cos \omega_{s} t & \cos \chi \sin \omega_{s} t & \frac{1}{2} \sin 2 \chi\left(1-\cos \omega_{s} t\right) \\ -\cos \chi \sin \omega_{s} t & \cos \omega_{s} t & \sin \chi \sin \omega_{s} t \\ \frac{1}{2} \sin 2 \chi\left(1-\cos \omega_{s} t\right) & -\sin \chi \sin \omega_{s} t & \cos ^{2} \chi+\sin ^{2} \chi \cos \omega_{s} t\end{array}\right) \mathbf{n}^{T}(0)$,

where $\omega_{s}=\sqrt{\omega_{0}^{2}+\left(\omega_{f, d}+\omega_{1}\right)^{2}}$ and $\chi=\arctan \left[\omega_{0} /\right.$ $\left.\left(\omega_{f, d}+\omega_{1}\right)\right]$ with $\omega_{0}=2 \mu B_{0}^{f, d} / \hbar$ and $\omega_{1}=2 \mu B_{z} / \hbar$. On the other hand, we can rewrite Eq. (5) clearly as

$$
\gamma_{\mathrm{AB}}^{\mathrm{eff}}(\mathbf{n}(0))=-\frac{\eta \omega_{f, d}}{2} \int_{0}^{2 \pi / \omega_{f, d}} \sin \theta \cos \left(\omega_{f, d} t-\varphi\right) d t
$$

with $\theta=\arctan \left(\sqrt{n_{1}^{2}+n_{2}^{2}} / n_{3}\right)$ and $\varphi=\arctan \left(n_{2} / n_{1}\right)$. Substituting Eq. (10) into Eqs. (6) and (11), the nonadiabatic noncyclic phases $\gamma_{\mathrm{AB}}^{\text {eff }}$ and $\gamma_{p}$ can be computed, at least numerically.

For simplicity, but without loss of generality, we compute $\widetilde{T}_{+}$and $\widetilde{T}_{-}$in a generalized Kronig-Penny ring consisting of $N=N_{+}+N_{-}$uniformly spaced $\delta$-function barriers with random strengths distributed uniformly in an interval $[-w / 2, w / 2]$, where $N_{+}\left(N_{-}\right)$is the number of barriers on the upper (lower) branch. In this model $\tilde{T}_{ \pm}$can be obtained analytically [22]. Substituting the obtained $\tilde{T}_{ \pm}$ and $\Delta \gamma$ into Eq. (9), we are able to calculate the transmission coefficient $T_{g}$.

In Fig. 1, we plot the calculated ensemble average Fourier spectrum of the conductance for unpolarized electrons, which is defined as

$$
\langle|G(\nu)|\rangle=\left\langle\left|\int_{-B_{m}}^{B_{m}} e^{i \nu B_{z}} G\left(B_{z}\right) d B_{z}\right|\right\rangle,
$$

where $G\left(B_{z}\right)=\left(e^{2} / h\right) \sum_{ \pm \mathbf{n}(0)} \bar{T}_{g}\left(B_{z}\right)$, with $\bar{T}_{g}$ the average on the initial spin orientation [23], and \langle\rangle represents the ensemble average. For reasonable comparison with the experimental observation [10], we choose parameters in the calculation as follows: $B_{m}=0.35 \mathrm{~T}$, $v_{f}=3.0 \times 10^{5} \mathrm{~m} / \mathrm{s}, \quad g=14, \quad r=1.05 \mu \mathrm{m} \quad$ (with $N_{ \pm}=4200$ ), which leads to the period in a magnetic field $\simeq 1.2 \mathrm{mT}$, and $w=0.267 k_{f}$, which corresponds to the mean free path $l \sim 1.0 \mu \mathrm{m}$ [24]. The dimensionless coefficient $\eta=3.5$, which corresponds to the SO coefficient $\hbar^{2} \kappa \sim 5.5 \times 10^{-10} \mathrm{eV} \mathrm{cm}$. Finally, it is typical to consider the case $\epsilon=0.25$. It is worth emphasizing that the essential feature of Fig. 1 is sensitive mainly to the SO coupling parameter $\eta$ : no clear splitting is present in the main peak if $\eta$ is smaller than about 1.5. This implies that the SO interaction plays a crucial role in the splitting. To understand the origin of the structure of the main peak, we plot it under both adiabatic and nonadiabatic conditions. From the inset in Fig. 1, we can see that under the adiabatic approximation a somewhat splitting of the main Fourier peak is present only if we include the effective $\mathrm{AB}$ phase. After careful analysis, we find that the effect of the adiabatic $\gamma_{p}$ phase seems too weak to play an important role in causing clearly observable splitting. More remarkably, if ever we take into account both the Pancharatnam phase and the effective $A B$ phase 


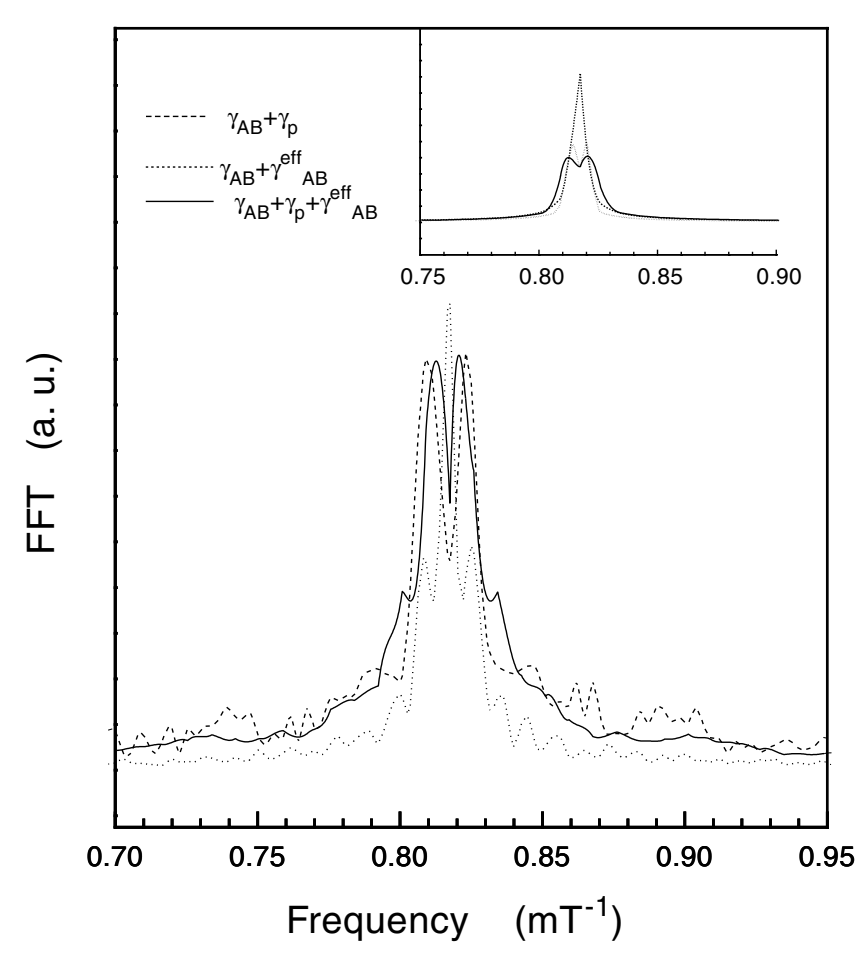

FIG. 1. The peak of the ensemble average Fourier spectrum of the conductance in nonadiabatic noncyclic cases. The inset shows the corresponding curves under the adiabatic condition.

in the nonadiabatic noncyclic case, as shown by the solid line in the main panel of Fig. 1, we observe clearly both the splitting and the side structure (two small peaks) of the main peak, which is in qualitative agreement with the experimental observation [10]. From Fig. 1, we can also see that the Pancharatnam phase plays a key role in the main splitting, while the two small side peaks are closely related to the effective $\mathrm{AB}$ phase. Therefore, we may conclude that the splitting of the main peak in the ensemble average Fourier spectrum stems from the nonadiabatic noncyclic Pancharatnam phase and the effective AB phase, both being dependent on the SO coupling.

Finally, we remark that a multichannel effect in the present quasi-1D ring, albeit weak and secondary, may exhibit in the ensemble average spectrum of the conductance (e.g., broadening and smearing of the peak splitting), which has been ignored and may deserve further study in the future.

We acknowledge support from a RGC grant of Hong Kong (HKU7118/00P). We also thank Professor P. K.
Mackeown for his critical reading of the manuscript.

*To whom correspondence should be addressed. Email address: zwang@hkucc.hku.hk

[1] Y. Aharonov and D. Bohm, Phys. Rev. 115, 485 (1959).

[2] M. V. Berry, Proc. R. Soc. London A 392, 45 (1984).

[3] Y. Aharonov and J. Anandan, Phys. Rev. Lett. 58, 1593 (1987).

[4] J. Samuel and R. Bhandari, Phys. Rev. Lett. 60, 2339 (1988).

[5] Y. Meir, Y. Gefen, and O. Entin-Wohlman, Phys. Rev. Lett. 63, 798 (1989).

[6] D. Loss and P. M. Goldbart, Phys. Rev. B 45, 13544 (1992).

[7] A. Stern, Phys. Rev. Lett. 68, 1022 (1992).

[8] A. G. Aronov and Y. B. Lyanda-Geller, Phys. Rev. Lett. 70, 343 (1993).

[9] T.Z. Qian and Z. B. Su, Phys. Rev. Lett. 72, 2311 (1994).

[10] A. F. Morpurgo et al., Phys. Rev. Lett. 80, 1050 (1998); Solid State Electron. 42, 1099 (1998).

[11] As pointed out in Ref. [12], although the magnetoresistance was experimentally measured, the statistical properties of conductance and resistance are the same, as we are interested in the metallic regime.

[12] A. G. Mal'shukov, V. V. Shlyapin, and K. A. Chao, Phys. Rev. B 60, R2161 (1999).

[13] Z. D. Wang and S. L. Zhu, Phys. Rev. B 60, 10668 (1999).

[14] C. N. Yang, Phys. Rev. Lett. 33, 7 (1974); T. T. Wu and C. N. Yang, Phys. Rev. D 12, 12 (1975).

[15] J. Anandan, Phys. Lett. A 138, 347 (1989).

[16] S. Oh and C. M. Ryu, Phys. Rev. B 51, 13441 (1995).

[17] Y. Aharonov et al., Phys. Rev. Lett. 73, 918 (1994).

[18] T. Choi et al., Phys. Rev. B 56, 4825 (1997).

[19] S. L. Zhu, Z. D. Wang, and Y. D. Zhang, Phys. Rev. B 61, 1142 (2000).

[20] M. Büttiker, Y. Imry, and M. Ya. Azbel, Phys. Rev. A 30, 1982 (1984).

[21] L. P. Lévy et al., Phys. Rev. Lett. 64, 2074 (1990); J. X. Zhu, Z. D. Wang, and Q. Wang, Z. Phys. B 102, 153 (1997).

[22] Z. S. Ma, H.Z. Li, and S. L. Zhu, Phys. Rev. B 53, 12597 (1996).

[23] Note that $\pm \mathbf{n}(t)$ just correspond to a pair of states with the initial spins of $\pm \mathbf{n}(0)$. This pair of states are always orthogonal in the present system. Moreover, we can prove that $\gamma_{p}(-\mathbf{n}(0))=-\gamma_{p}(\mathbf{n}(0))$ and $\gamma_{\mathrm{AB}}^{\text {eff }}(-\mathbf{n}(0))=$ $-\gamma_{\mathrm{AB}}^{\text {eff }}(\mathbf{n}(0))$, a fact which is in principle responsible for the splitting of the main peak.

[24] C. M. Soukoulis et al., Phys. Rev. Lett. 50, 764 (1983). 